\title{
A Comparison of BPMN and UML 2.0 Activity Diagrams
}

\author{
Daniela C. C. Peixoto ${ }^{1}$, Vitor A. Batista ${ }^{1}$, Ana P. Atayde ${ }^{1}$, Eduardo P. Borges ${ }^{1}$, \\ Rodolfo F. Resende ${ }^{2}$, Clarindo Isaías P. S. Pádua ${ }^{1}$. \\ ${ }^{1}$ Synergia - Universidade Federal de Minas Gerais \\ ${ }^{2}$ Departamento de Ciência da Computação - Universidade Federal de Minas Gerais \\ Av. Antônio Carlos 6627 - CEP 31270-010 - Belo Horizonte - MG - Brasil \\ \{cascini, vitor, atayde, eborges, rodolfo, clarindo\}@dcc.ufmg.br
}

\begin{abstract}
Interest in evaluating Business Process Modeling Languages has widespread, in part due to the increase of the number of languages available for this purpose. Several works on the evaluation of BPMLs are available. Their evaluation are mainly based on perspectives centered in modeling experts. In this paper, we address the readability perspective of two BPMLs (UML 2.0 and $B P M N)$ for people not familiar with process modeling.
\end{abstract}

The UML can be tailored for purposes beyond software modeling and offers Activity Diagrams for business process modeling. BPMN was designed for modeling business process and has a primary goal of being understandable by all business stakeholders. We compared undergraduates (freshmen) understanding of business process modeled in BPMN and UML 2.0 Activity Diagrams. Our results are interesting, since we were able to find that these two languages do not have significant differences, despite BPMN readability design goals.

\section{Introduction}

A business process modeling focus on describing activities within the business and how they interact with the resources in the business to achieve a goal. According to Penker [Eriksson $\&$ Penker, 2000] a business process model may have six different reasons to be created, which are: to understand the key mechanisms of an existing business; to orient the creation of suitable information systems that support the business; to implement improvements in the current business; to show the structure of an innovated business; to experiment new business concepts; and to identify business elements not considered part of the core, which could be delegated to an outside supplier.

The increasing interest in business process modeling has resulted in the appearance of various Business Process Modeling Languages (BPMLs). Today, there are several notations that can be used for business process modeling [List \& Korherr, 2006]: UML 2.0 Activity Diagram [OMG, 2005], Business Process Modeling Notation [OMG, 2006], Event Driven Process Chain (EPC) [Scheer, 1999], Integrated DEFinition Method 3 (IDEF3) [Mayer \& Perakath, 1995], and others. These languages express certain aspects of processes, for example, activities and roles, and address different application areas. However 
no one of these languages is predominant in the business process modeling area [Mendling et al., 2004]. One major reason [zur Muehlen, 2004] is the wide disparity in the needs and viewpoints of various modelers and designers.

There are many different stakeholders interested in the results of a business process modeling. They correspond to people that participate in the process directly or indirectly as the owner, external customers (example: business modelers or designers) and internal customers (example: managers or employees). The owner sets the goals and allocates the resources to make the business run. The business modeler describes the processes using specific notations and tools [Eriksson \& Penker, 2000]. It is important to note that the internal business customers do not need to be an expert in these BPMLs, they only need to understand the results of the modeling, more specifically, and they should know how to read business process diagrams.

Before the selection of an appropriate business modeling language, the modelers and designers should observe the kind of process being modeled and the purpose for which the modeling is being done [Russell et al., 2006b].

This article investigates two BPMLs - UML 2.0 Activity Diagrams (AD) and BPMN - capacity of being readily understood. Our main contribution consists of an experiment with computer science students (freshmen) representing internal customers reading business process diagrams.

The Unified Modeling Language (UML) [OMG, 2005] is increasingly being seen as a de facto standard for software modeling and design. UML is an object-oriented notation that embodies a set of formalisms for capturing detailed design aspects of software systems. The UML can be tailored for several purposes and its Activity Diagram can be used for business process modeling [Wohed et al., 2005].

Business Process Modeling Notation (BPMN) was designed for modeling business process and their transformation into an execution language. The primary goal of the BPMN is to be understandable by all business stakeholders [OMG, 2006].

We originally expected that BPMN models were easier to understand than UML 2.0. This assumption was derived from the fact that BPMN primary goal was to be understandable by all business stakeholders. Furthermore, BPMN has model elements that, in some cases, do not have a correspondent element in UML 2.0 AD.

The comparison in our work is based on Workflow Patterns, a taxonomy of generic, recurring constructs originally devised to evaluate workflow systems, and more recently used to successfully evaluate workflow standards, business process languages and processaware information systems in general [White, 2004], [Wohed et al., 2003].

The remainder of this paper is structured as follows. Section 2 presents some works that describe evaluations of BPMLs. Section 3 presents the workflow patterns. Section 4 describes the methodology used for comparing UML 2.0 AD e BPML. Section 5, 6 and 7 reports the results achieved. Section 8 concludes and suggests future work. 


\section{Related Work}

In BPML related works the evaluated concepts are mainly based on meta-models, which address a very technical perspective.

List and Korherr [List \& Korherr, 2006], for example, propose a generic meta-model for evaluating BPMLs. This meta-model is categorized using five perspectives, inspired by business process theory [Ould, 1995], Workflow Patterns [Russell et al., 2006a], and the Workflow Management Coalition (WfMC) [WMC, 1998]:

- Organizational: represents where and by whom (agents) process elements are performed;

- Functional: represents the process elements which are being performed, e.g., activities;

- Behavioral: represents when and how process elements are performed;

- Informational: represents the informational entities produced or manipulated by a process; and

- Business process context: provides an overview perspective of the process and describes major business process characteristics, such as goals.

List and Korherr evaluated seven BPMLs, including UML2.0 Activity Diagram [OMG, 2005], Business Process Modeling Notation (BPMN) [OMG, 2006], Business Process Definition Metamodel (BPDM) [OMG, 2004], Role Activity Diagram (RAD) [Holt \& Grimes, 1983], Event Driven Process Chain (EPC) [Scheer, 1999], and Petri Net [Gou et al., 2000]. They observed that the functional and the behavioral perspectives are very well represented in all BMPLs, while the organizational and informational perspectives are only partly supported, and business process context is not explicitly supported.

Russell examines the suitability of UML 2.0 Activity Diagrams for business process modeling, using Workflow Patterns [Russell et al., 2006b] as an evaluation framework. The pattern evaluation shows that UML 2.0 Activity Diagrams is not suitable for representing all aspects of this type of modeling. It offers support for control-flow and data perspective allowing most of the constructs to be directly captured. However, it is extremely limited in modeling resource-related or organizational aspects of business process. These limitations are shared with most of the other BPMLs, showing the emphasis that has been placed on the control-flow and data perspectives in these notations.

White [White, 2004] examines whether two modeling notation, BPMN and UML 2.0 Activity Diagrams, can graphically represent the control flow workflow patterns. White examined 21 workflow patterns and observed that they could adequately model most of them. Both notations provide similar solutions for most of the patterns indicating how close the notation is in their presentation. The similarities in the two diagrams are explained by the fact that both were designed to solve the problem of the diagramming of procedural business processes. Some differences are due to the fact that they have different target users of diagrams: business people for BPMN, and software developer for UML 2.0 (this language is more technically oriented).

It is interesting to observe that in all these evaluations, characteristics related to the consumption of business modeling results (legibility, for example) are not addressed at all. 


\section{Workflow Patterns}

Russel et al [Russell et al., 2006a] identify patterns in four perspectives ${ }^{1}$ : control-flow, data, resource and exception handling. The control-flow perspective captures aspects related to control-flow dependencies. Originally twenty patterns were proposed for this perspective, but in the latest revision this has grown to forty-two patterns. This perspective includes patterns for Basic Control Flow, Advanced Branching and Synchronization, Multiple Instance, Cancellation and Force Completion, Iteration, Termination and Trigger The data perspective represents the definition and use of data elements (visibility), information passing, data transfer and interaction with other perspectives. This perspective includes patterns for Data visibility, Data Interaction (Internal Data Interaction and External Data Interaction), Data Transfer Mechanisms and Data-based Routing. The resource perspective captures the various ways in which resources are represented and utilized in workflows. This perspective includes patterns for Creation, Push, Pull, Detour, Auto-Start, Visibility, and Multiple Resource. Finally the patterns for the exception handling perspective deal with the various causes and the resultants actions need to be taken as a result of exception occurrences. All these patterns range from very simple to very complex aspects of business process models.

\section{The experiment}

In this section, we described the techniques used to analyze UML 2.0 $\mathrm{AD}$ and BPMN related to the legibility of the business process model. We have conducted a controlled experiment to examine this characteristic based on end-users point of view. Our strategies follow the experiment process defined by Wohlin et al. [Wohlin et al., 2000] that consists of five activities: definition, planning, operation, analysis \& interpretation, and presentation $\&$ package.

With this experiment we evaluated the reading activity of a notation, which is the process of parsing a notation and creating an understanding of it [Wright \& Cockburn, 2005]. We do not evaluate other tasks executed when modeling business process, e.g., writing.

\subsection{Hypothesis}

This evaluation tests the hypothesis that using a BPMN notation for a business process model is easier to understanding than using UML 2.0 AD notation.

\footnotetext{
${ }^{1}$ See http://www.workflowpatterns.com/patterns/index.php for more details.
} 


\subsection{Experiment details}

The experiment was conducted with 35 undergraduate computer science students. All participants were in the first year of this course. During this experiment, these students represent internal customers, so they need to read and understand business process diagrams. At the experiment beginning, the instructor explained to the participants what they were expected to do during that experiment. Firstly, the participants answered a questionnaire with the purpose of collecting their knowledge about the subject under study: business process domain (a retirement process) and BPMNs. After that, the students were randomly assigned to one version of the diagrams (BPMN or in UML 2.0 AD). Every student saw four diagrams and answered 11 true/false questions related to the semantics of the diagrams.

The diagrams modeled government retirement process. The diagrams were selected from models produced in three months by a team of five experienced business process modelers. These models were produced with the aim to document and guide the improvement of the organization. We decided to choose diagrams from real project since there is no business process models database available to be used as a benchmark.

Figure 1 shows an example of BPMN diagram used in the experiment. Figure 2 shows the same diagram modeled in UML 2.0.

The diagrams were organized in two levels of abstraction. The first level shows the main processes and the second level shows sub-processes details. The diagrams were available in HTML format and the students could navigate through all levels of these diagrams. Both notations were implemented in Sparx Enterprise Architect tool and they were modeled, in both languages, in a very similar way. Whenever possible, processes, objects and other elements of these notations were equally arranged.

The diagrams were chosen trying to maximize usage of the following workflow patterns. These patterns summarize the findings presented in [Russell et al., 2006a], thus serving as a guide for the evaluation of BPMN and UML 2.0 AD.

- WCP-1: Sequence - the ability to depict a sequence of activities, with one activity starting after a previous activity has completed;

- WCP-2: Parallel split - the ability to execute activities concurrently;

- WCP-3: Synchronization - the ability to capture a convergence of two or more branches, generated by the Parallel split pattern, into a single subsequent branch;

- WCP-4: Exclusive choice - the ability to represent a decision point where only one of the branches is chosen;

- WCP-5: Simple merge - the ability to show the convergence of two or more branches into one, without synchronization.

- WCP-6: Multi-choice - the ability to depict a divergence of a branch into two or more parallel branches on a selective bases;

- WCP-10: Arbitrary cycles - the ability to represent loops in a process that have more than one entry or exit point.

WCP means Workflow Control Pattern. Each question was assigned to a main goal, that is, each question was assigned to the workflow pattern(s) related to it. This assignment 
was helpful in order to map the use of each pattern in the diagrams. Table 1 illustrates this relation.

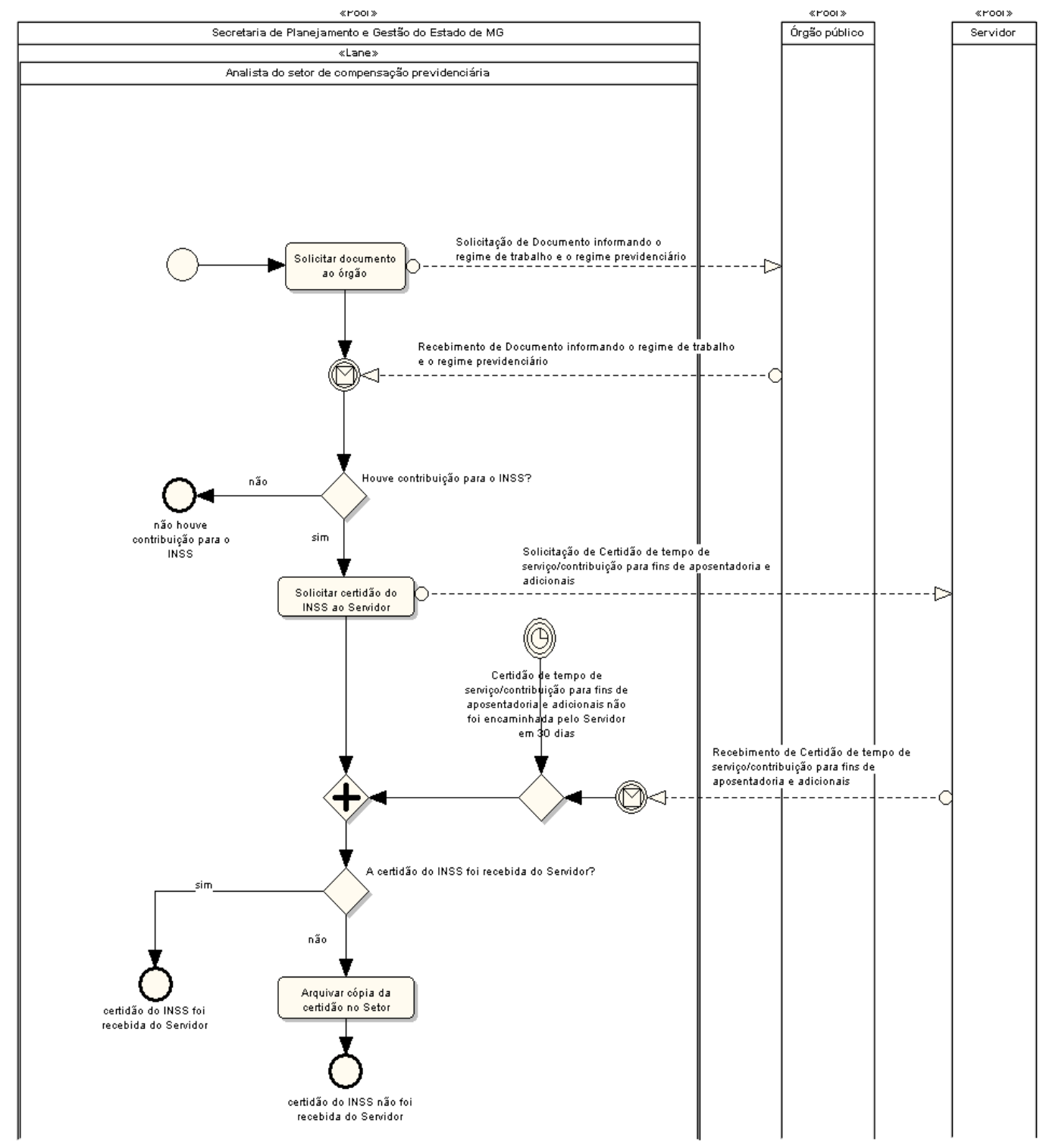

Figure 1 - A BPMN diagram used in the experiment

An example of a question that evaluates Arbitrary Cycles and Multiple choice (Question number 10 - Table 1) is "The activity Sanar Irregularidades could be executed several times during the whole process execution. True or False?". Figure 3 shows an excerpt from a diagram related to this question.

Participants were given as much time as needed to complete the task and the instructor collected the questionnaires with the answers after they have finished. 


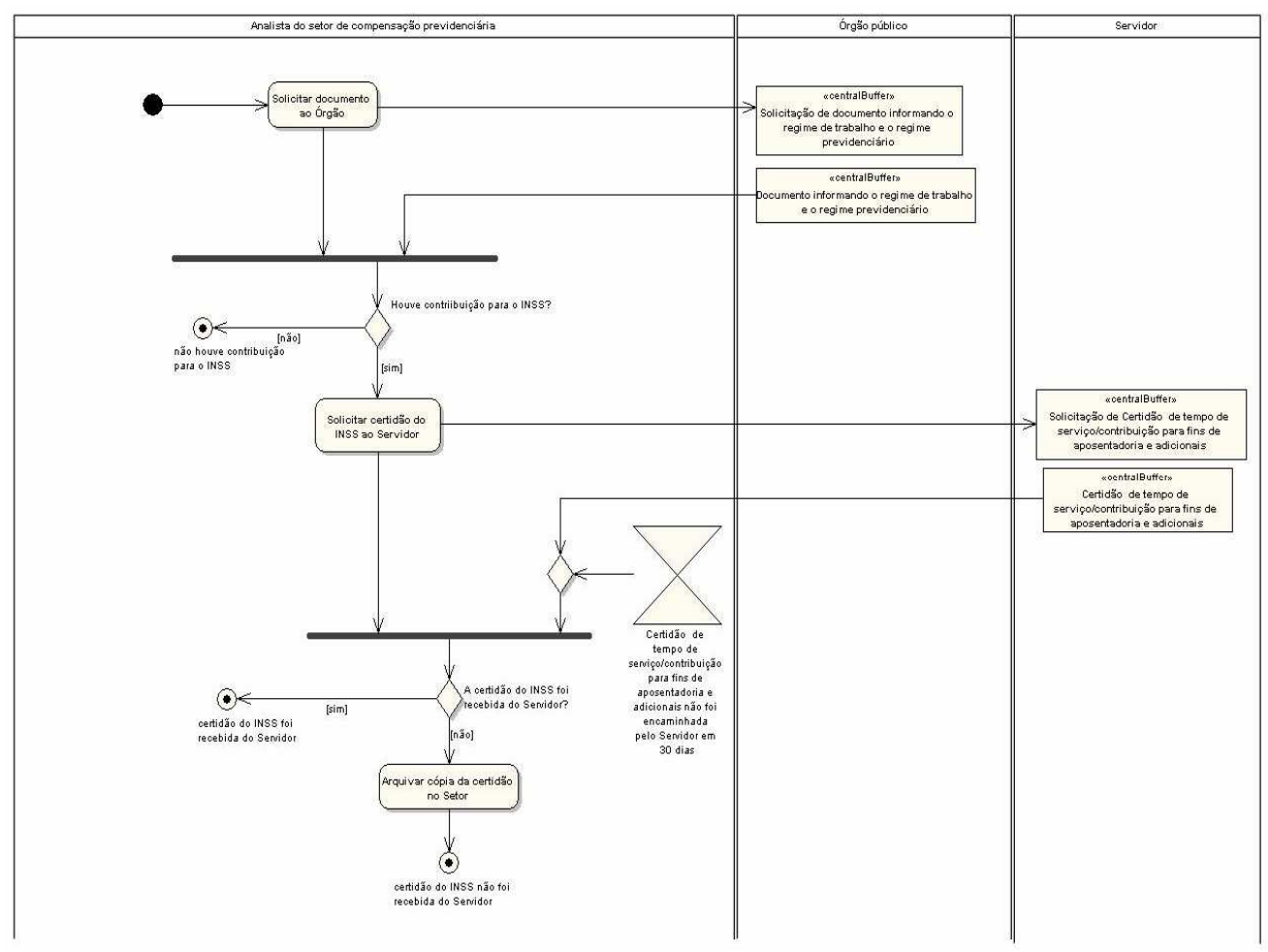

Figure 2 - UML 2.0 diagram used in the experiment.

Table 1 - Purpose of each question and patterns used

\begin{tabular}{|c|l|l|}
\hline Question & \multicolumn{1}{|c|}{ What was evalutated } & \multicolumn{1}{|c|}{ Patterns used } \\
\hline 1 & Simple activities sequence & Sequence \\
\hline 2 & Branch after decisions & $\begin{array}{l}\text { Sequence, Exclusive } \\
\text { Choice }\end{array}$ \\
\hline 3 & Use of artifacts and decisions & $\begin{array}{l}\text { Sequence (multiple } \\
\text { diagrams), Exclusive } \\
\text { Choice }\end{array}$ \\
\hline 4 & Roles and multiple diagrams & \\
\hline 5 & Roles and decisions & $\begin{array}{l}\text { Sequence (multiple } \\
\text { diagrams), Exclusive } \\
\text { Choice, Simple Merge }\end{array}$ \\
\hline 6 & Branch after decisions & $\begin{array}{l}\text { Sequence, Exclusive } \\
\text { Choice, Simple Merge }\end{array}$ \\
\hline 7 & Parallelism and synchronization & Synchronization \\
\hline 8 & Roles & $\begin{array}{l}\text { Sequence (multiple } \\
\text { diagrams), Simple } \\
\text { Merge }\end{array}$ \\
\hline 9 & Decisions over multiple \\
diagrams & $\begin{array}{l}\text { Multiple choice, Arbitrary } \\
\text { Cycles }\end{array}$ \\
\hline 10 & Loops over multiple diagramion \\
\hline 11 & Messages between roles & Synchronization \\
\hline
\end{tabular}




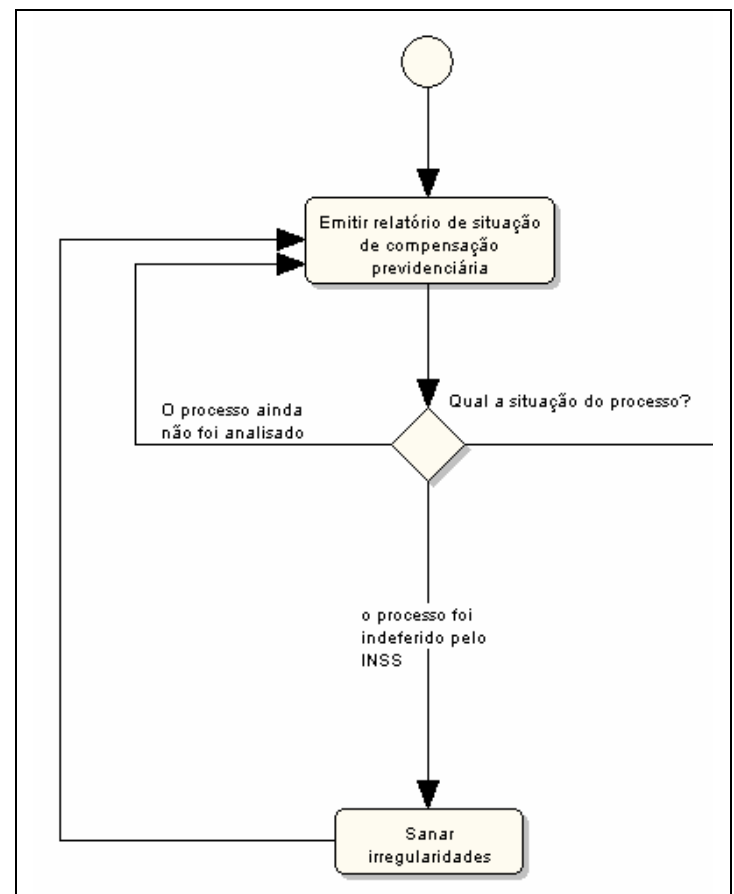

Figure 3 - Excerpt of diagram used in the experiment to evaluate arbitrary cycles

\section{Preliminary Results}

According to profile forms, no students have had experience with any of these notations before or with the kind of business that was modeled in the diagrams (government retirement process). To examine the hypothesis stated we used Mann-Whitney test [Triola, 2008] - since data is not normally distributed - to compare the scores on each question and the total score for both questionnaires. Table 2 shows the global score for each notation. This score was calculated dividing the number of correct answers by the number of questions (11 in this case). Table 3 shows a per question comparison using proportion of correct answers. All results presented in this paper have $95 \%$ of confidence.

These tables show an interesting pattern of results. The number of correct answers obtained in both languages is very similar.

Table 2 - Global results: percentage of correct answers.

\begin{tabular}{|c|c|c|c|}
\hline Notation & Forms & Mean & StDev \\
\hline UML & 17 & 8.412 & 1.46 \\
\hline BPMN & 18 & 9.444 & 1.423 \\
\hline
\end{tabular}


Table 3 - Correct answers per question

Comparison of proportion of correct answers between notations

Note that UML has 17 forms and BPMN 18

\begin{tabular}{|c|c|c|c|c|c|}
\hline \multirow[b]{2}{*}{ Question } & \multicolumn{2}{|c|}{ Correct answers } & \multirow{2}{*}{$\begin{array}{l}\text { Proportion } \\
\text { Difference }\end{array}$} & \multirow{2}{*}{$\begin{array}{l}\text { Interval of } \\
\text { proportion } \\
\text { difference }\end{array}$} & \multirow[b]{2}{*}{ P-value } \\
\hline & UML & BPMN & & & \\
\hline 1 & 13 & 18 & 0.235 & $(0,033 ; 0,436)$ & 0.022 \\
\hline 2 & 15 & 16 & 0.007 & $(-0,204 ; 0,217)$ & 0.952 \\
\hline 3 & 10 & 13 & 0.134 & $(-0,178 ; 0,446)$ & 0.400 \\
\hline 4 & 11 & 16 & 0.242 & $(-0,027 ; 0,511)$ & 0.079 \\
\hline 5 & 8 & 13 & 0.252 & $(-0,063 ; 0,566)$ & 0.117 \\
\hline 6 & 15 & 17 & 0.062 & $(-0,124 ; 0,248)$ & 0.513 \\
\hline 7 & 16 & 13 & -0.219 & $(-0,454 ; 0,016)$ & 0.068 \\
\hline 8 & 12 & 16 & 0.183 & $(-0,077 ; 0,443)$ & 0.169 \\
\hline 9 & 14 & 16 & 0.065 & $(-0,166 ; 0,297)$ & 0.581 \\
\hline 10 & 15 & 14 & -0.105 & $(-0,350 ; 0,141)$ & 0.404 \\
\hline 11 & 14 & 18 & 0.176 & $(-0,004 ; 0,357)$ & 0.056 \\
\hline
\end{tabular}

Table 3 shows that UML questions were easier for students to answer in only 2 of 11 questions. However, only question number 1 had a significant difference between notations (note that in all other questions, the confidence interval of difference includes zero).

\subsection{Time}

Mann-Whitney test did not show that the task in one notation was completed faster than in the other one. Participants needed a mean of $15.89 \pm 2.11$ minutes to answer the questionnaire in BPMN and a mean of $16.37 \pm 2.96$ minutes to answer the same questionnaire in UML 2.0 AD.

\subsection{Hit rate}

Mann-Whitney test also did not show any differences in the results related to hit rate of the answers. Participants achieved a mean of 9.44 correct answers of 11 in BPMN and a mean of 8.41 in the same questionnaire in UML 2.0 AD. Figure 4 shows the interval plot of the results presented in Table 2 . Observe the overlapped confidence intervals.

\section{Comments from Participants}

After taking part, participants were asked about the clarity of the questions. General comments: $6 \%$ of the participants commented the questions were hard, and they needed some training for doing better. 68\% said they had some difficult in understanding the questions, but they were able to do it, and $26 \%$ thought the questions were easy and could understand them with no major problems. 
- UML 2.0 AD: four of seventeen participants dealing with UML 2.0 AD diagrams found that it was easy to answer the questions, 12 found difficult, but not too much, and one found it was very hard to answer the questions.

- $\quad$ BPMN: five of eighteen participants dealing with the BPMN diagrams found easy to answer the questions, 12 found it that was difficult, but not too much, and one found it was very hard to answer the questions.

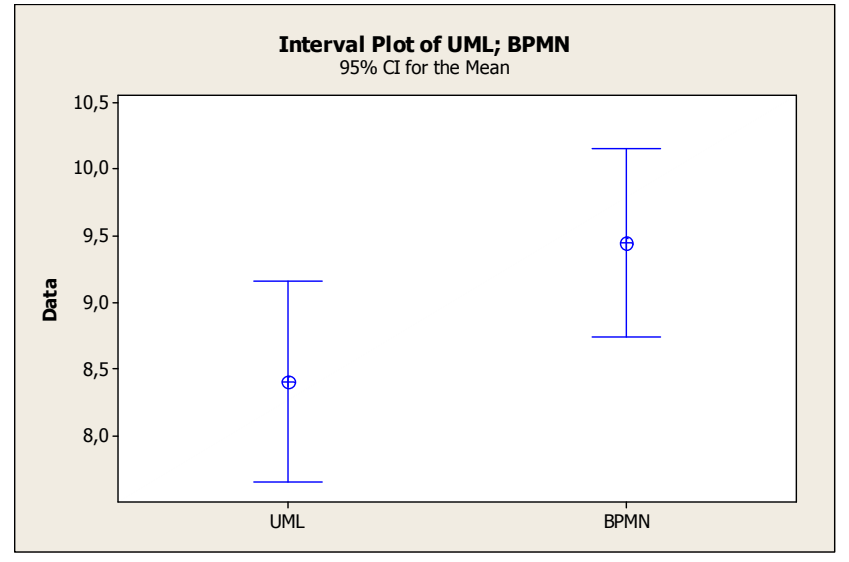

Figure 4 - Hit rate of answers

\section{Additional Comments}

With these results, we could not confirm that exist differences in the business modeling using BPMN and UML 2.0 Activity Diagrams from the point of view of end user readability. However, in our study we have analyzed only workflow patterns described in Table 1 . This suggests that, as far as these patterns are concerned, the level of difficult for understanding the business process, in both languages, is the same. Our proposed hypothesis that using a BPMN notation for a business process model is easier to understanding than using UML 2.0 $\mathrm{AD}$ notation was rejected.

\section{Conclusions and future work}

This paper describes an evaluation examining the readability of business models written in BPMN and UML 2.0 AD. We used computer science freshmen not familiar with the languages and with the modeled domain, representing internal customers of one organization.

We originally expected that BPMN models were easier to understand than UML 2.0 AD. This assumption was derived from the BPMN primary goal and its distinct model elements not present in UML 2.0 AD.

Our study does not offer evidence that exist differences in modeling using BPMN and UML 2.0 Activity Diagrams from the point of view of end user readability. Indeed, we 
hope that these results provide more information to organizations in deciding to adopt or not a new notation for business process modeling. In our study we have analyzed only workflow patterns described in Table 1. This suggests that using these patterns the level of difficult for understanding the business process, in both languages, is the same.

This restriction is an important scope for future work. It is important to determine whether there are differences in the results using other workflow patterns. It is also important to determine if there are differences in other modeling activities like model writing. Other research questions include:

- Is this lack of difference due to a limited use of workflow patterns? To answer this question we need to run experiments using other workflow patterns. Re-running this evaluation will tell us with a greater level of confidence the differences between these notations.

- Does the difference exist on the writing activity? To answer this question we need to run evaluations concentrating on the writing activities. This evaluation will give us a base to examine the effect of each notation.

\section{References}

[Eriksson \& Penker, 2000] Eriksson, H., \& Penker, M. 2000. Business Modeling with UML: business patterns at work. John Wiley \& Sons.

[Gou et al., 2000] Gou, H., Huang, B., Liu, W., Ren, S., \& Y., Li. 2000. Petri-net-based business process modeling for virtual enterprises Systems. Pages 3183 - 3188 of: IEEE International Conference on Man, and Cybernetics, 2000, vol. 5.

[Holt \& Grimes, 1983] Holt, A, Ramsey R., \& Grimes, J. 1983. Coordinating System Technology as the Basis for a Programming Environment. Electrical Communication, 57(4), $307-314$.

[List \& Korherr, 2006] List, Beate, \& Korherr, Birgit. 2006. An evaluation of conceptual business process modelling languages. Pages 1532-1539 of: SAC '06: Proceedings of the 2006 ACM symposium on Applied computing. New York, NY, USA: ACM Press.

[Mayer \& Perakath, 1995] Mayer, R., Menzel C. Painter M. Witte P. Blinn T., \& Perakath, B. 1995. Information Integration for Concurrent Engineering (IICE) IDEF3 Process Description Capture Method Report. Tech. rept. Knowledge Based Systems Inc. (KBSI).

[Mendling et al., 2004] Mendling, J., uttgens, N., \& Neumann, M. 2004. A Comparison of XML Interchange Formats for Business Process Modelling.

[OMG, 2004] OMG. 2004 (January). Business Process Definition Metamodel - Version 1.0.2.

[OMG, 2005] OMG. 2005. Unified Modeling Language Specification, version 2.0. Object Management Group (OMG). 
[OMG, 2006] OMG. 2006. Business Process Modeling Notation Specification. Object Modeling Group.

[Ould, 1995] Ould, M. 1995. Business Process - Modeling and Analysis for Reengineering and Improvement. John Wiley \& Sons.

[Russell et al., 2006a] Russell, N., ter Hofsted, A.H.M., van der Aalst, W.M.P., \& Mulyar, N. 2006a. Workflow Control-Flow Patterns: A Revised View. Tech. rept. BPM Center Report BPM-06-22.

[Russell et al., 2006b] Russell, Nick, van der Aalst, Wil M. P., ter Hofstede, Arthur H. M., \& Wohed, Petia. 2006b. On the suitability of UML 2.0 activity diagrams for business process modelling. Pages 95-104 of: APCCM '06: Proceedings of the 3rd Asia-Pacific conference on Conceptual modelling. Darlinghurst, Australia, Australia: Australian Computer Society, Inc.

[Scheer, 1999] Scheer, A. W. 1999. ARIS - Business Process Modeling. 2nd edition edn. Springer Verlag.

[Triola, 2008] Triola, Mario F. 2008. Introdução à Estatística. 10a. edição edn. LTC Editora.

[White, 2004] White, Stephen A. 2004. Process Modeling Notations and Workflow Patterns.

[WMC, 1998] WMC. 1998. Workflow Management Coalition. Interface 1: Process Definition Interchange Process Model, WfMC TC-1016-M (1998).

[Wohed et al., 2003] Wohed, P., Perjons, E., Dumas, M., \& ter, A. 2003. Pattern based analysis of EAI languages - the case of the business modeling language.

[Wohed et al., 2005] Wohed, Petia, van der Aalst, Wil M.P., Dumas, Marlon, ter Hofstede, Arthur H.M., \& Russell, Nick. 2005. Pattern-based analysis of the control flow perspective of UML activity diagrams. In: ER 2005: 24th International Conference on Conceptual Modelling. Springer Verlag.

[Wohlin et al., 2000] Wohlin, Claes, Runeson, Per, Höst, Martin, Ohlsson, Magnus C., Regnell, Björn, \& Wesslén, Anders. 2000. Experimentation in Software Engineering, An Introduction. Kluwer Academic Publishers.

[Wright \& Cockburn, 2005] Wright, Tim, \& Cockburn, Andy. 2005. Evaluation of two textual programming notations for children. Pages 55-62 of: AUIC '05: Proceedings of the Sixth Australasian conference on User interface. Darlinghurst, Australia, Australia: Australian Computer Society, Inc.

[zur Muehlen, 2004] zur Muehlen, M.; Rosemann, M. 2004. Multi-Paradigm Process Management. Pages 169-175 of: Janis Grundspenkis, Marite Kirikova, Riga Latvia (ed), Proceedings of CAiSE'04 Workshops - 5th Workshop on Business Process Modeling, Development and Support (BPMDS 2004). 